\title{
ECG Rhythm Analysis During Manual Chest Compressions Using an Artefact Removal Filter and Random Forest Classifiers
}

\author{
Iraia Isasi ${ }^{1}$, Ali Bahrami Rad ${ }^{2}$, Unai Irusta ${ }^{1}$, Morteza Zabihi $^{3}$, Elisabete Aramendi ${ }^{1}$, Trygve Eftest $ø 1^{4}$, \\ Jo Kramer-Johansen ${ }^{5}$, Lars Wik ${ }^{5}$ \\ ${ }^{1}$ University of the Basque Country (UPV/EHU), Bilbao, Spain \\ ${ }^{2}$ Aalto University, Espoo, Finland \\ ${ }^{3}$ University of Technology, Tampere, Finland \\ ${ }^{4}$ University of Stavanger, Stavanger, Norway \\ ${ }^{5}$ Oslo University Hospital, Oslo, Norway
}

\begin{abstract}
Interruptions in cardiopulmonary resuscitation $(C P R)$ decrease the chances of survival. However, CPR must be interrupted for a reliable rhythm analysis because chest compressions (CCs) induce artifacts in the ECG. This paper introduces a double-stage shock advice algorithm (SAA) for a reliable rhythm analysis during manual CCs. The method used two configurations of the recursive least-squares $(R L S)$ filter to remove CC artifacts from the ECG. For each filtered ECG segment over 200 shock/no-shock decision features were computed and fed into a random forest (RF) classifier to select the most discriminative 25 features. The proposed $S A A$ is an ensemble of two RF classifiers which were trained using the 25 features derived from different filter configurations. Then, the average value of class posterior probabilities was used to make a final shock/no-shock decision. The dataset was comprised of 506 shockable and 1697 non-shockable rhythms which were labelled by expert rhythm resuscitation reviewers in artifact-free intervals. Shock/no-shock diagnoses obtained through the proposed double-stage SAA were compared with the rhythm annotations to obtain the Sensitivity (Se), Specificity ( $S p)$ and balanced accuracy (BAC) of the method. The results were 93.5\%, 96.5\% and 95.0\%, respectively.
\end{abstract}

\section{Introduction}

Minimum "hands-of" intervals during cardiopulmonary resuscitation (CPR) are required to improve the chances of a successful defibrillation [1]. In current practice CPR is interrupted every 2 minutes for a reliable analysis of the heart rhythm. In fact, chest compressions (CCs) provided during CPR induce artifacts in the ECG that impede a reliable rhythm analysis of shock advice algorithms.

Over the last 15 years, many efforts have been made to achieve a continuous rhythm analysis without interruptions to CPR therapy. Different approaches have been proposed, such as rhythm analysis during ventilation pauses $[2,3]$, however the main approach has been designing adaptive filters to suppress the artifact and then diagnose using a SAA for artifact-free ECG [4]. Nevertheless, the accuracy of this approach is still poor. Adaptive filters substantially reduce CC artifacts with high SNR improvements, however filtering residuals frequently resemble a disorganized rhythm. In these cases, SAAs may produce a wrong shock diagnosis as the majority of the SAAs used are designed for artifact-free ECGs. This is the reason why current methods have a high capacity to detect shockable rhythms, Sensitivity (Se), but a low capacity to detect non-shockable rhythms, Specificity (Sp).

Recently, a multistage algorithm was introduced to increase the Sp [5] (supp materials). In brief, this algorithm uses two recursive least squares (RLS) filters and a SAA of a commercial defibrillator in three decision stages. Although this solution considerably improves the Sp of previous approaches, it still does not meet American Heart Association's criteria for a reliable rhythm diagnosis (Sp $>95 \%, \quad S e>90 \%)$ during manual CCs. Another approach to increase the $\mathrm{Sp}$ was the use of machine learning techniques to classify the ECG after using an adaptive CPR artifact suppression filter [6].

In this paper, we propose a method for a reliable shock advise during manual $\mathrm{CCs}$, which combines the both aforementioned approaches: a double stage RLS filtering [5] and a SAA algorithm based on random forest (RF) classifiers [6] which benefits from both filtering configurations to reach a reliable shock/no-shock decision. 


\section{Materials and methods}

\subsection{Dataset}

The data were obtained from a prospective study of out-of-hospital cardiac arrest (OHCA) patients gathered between March 2002 and September 2004 by the emergency services of London, Stockholm and Akershus and coordinated by the Oslo University Hospital. The ECG and the compression depth (CD) signals were acquired using a modified version of Laerdal's Heartstart 4000 defibrillator (4000SP) and were resampled to $250 \mathrm{~Hz}$. A notch and a Hample filter were used to remove $50 \mathrm{~Hz}$ noise and spiky artifacts from the ECG, respectively. Finally, the ECG was band limited to $0.5-40 \mathrm{~Hz}$. CC instants $\left(\mathrm{t}_{\mathrm{k}}\right)$ were automatically marked in the $\mathrm{CD}$ signal using a negative peak detector for depths above $1 \mathrm{~cm}$, see figure 1 .

The dataset used in this study contained 2203 records obtained from 273 OHCA patients. Each record (see figure 1) consisted of two consecutive intervals: a 15-sec interval which includes continuous CCs, and a 5-sec interval free of artifact. The latter interval was reviewed by expert resuscitation rhythm reviewers to annotate the patient's underlying rhythm as shock/no-shock and use it as ground truth. In total there were 506 shockable and 1697 non-shockable rhythms.

\subsection{Filtering the $\mathrm{CC}$ Artifact}

In this work, the used CC artefact suppression method is based on a recursive least squares (RLS) filter adapted to estimate periodic interferences [5]. The RLS filter estimates the time-varying coefficients $\left(a_{k}(n)\right.$ and $\left.b_{k}(n)\right)$ of a multiharmonic model of the artifact whose fundamental frequency $\left(f_{0}(n)\right)$ is derived from the chest compression instants $\left(t_{k}\right)$ :

$$
\begin{gathered}
s_{\mathrm{cc}}(n)=\sum_{k=1}^{N} a_{k}(n) \cos \left(k 2 \pi f_{0}(n) n T_{s}\right)+ \\
b_{k}(n) \sin \left(k 2 \pi f_{0}(n) n T_{s}\right) \\
f_{0}(n)=\frac{1}{t_{k}-t_{k-1}} \quad t_{k-1}<n T_{s} \leq t_{k}
\end{gathered}
$$

The CC artifact is iteratively estimated $\left(\hat{s}_{\mathrm{cc}}\right)$ and subtracted from the corrupted ECG ( $\left.s_{\text {cor }}\right)$, to obtain the clean ECG $\left(\hat{s}_{\mathrm{ecg}}\right)$, as shown in figure 1 .

In the RLS filter there are two degrees of freedom, the number of harmonics needed to model the artifact $(N)$ and the forgetting factor $(\lambda)$ which controls the coarseness of the filter. In this paper, the corrupted ECG was filtered for two configurations of the RLS filter $(N / \lambda)$ following the optimal configuration of the multistage algorithm described in [5] for manual CCs. In the first stage, the corrupted ECG was coarsely filtered $\left(\hat{s}_{\operatorname{ecg} \lambda_{1}}\right)$ using a $\lambda$ of 0.987 whereas in the second stage the ECG was finely filtered $\left(\hat{s}_{\operatorname{ecg} \lambda_{2}}\right)$ with a $\lambda$ fixed to 0.998 . In both stages $N$ was set to 4 .

\subsection{Feature engineering}

For each filtered ECG $\left(\hat{s}_{\operatorname{ecg} \lambda_{1}}, \hat{s}_{\operatorname{ecg} \lambda_{2}}\right)$, a multi-resolution analysis is employed to extract 244 features. Only the interval from $4 \mathrm{~s}$ to $12 \mathrm{~s}$ was used to compute features. First $4 \mathrm{~s}$ were left out to avoid RLS filtering transients. The 8-second ECG segments were decomposed by discrete wavelet transform (DWT) into its subbands with the Daubechies 4 wavelet and 7 levels of decomposition generating a set of approximation coefficients $a_{7}$ and seven sets of detail coefficients $d_{1}$ to $d_{7}$. The ECG was then reconstructed, $s(n)$, by using detail coefficients $d_{3}-d_{7}$. Reconstructed signals corresponding to each set of detail coefficients $\left(d_{3}\right.$ to $\left.d_{7}\right)$ were also generated: $s_{3}(n)$ to $s_{7}(n)$.

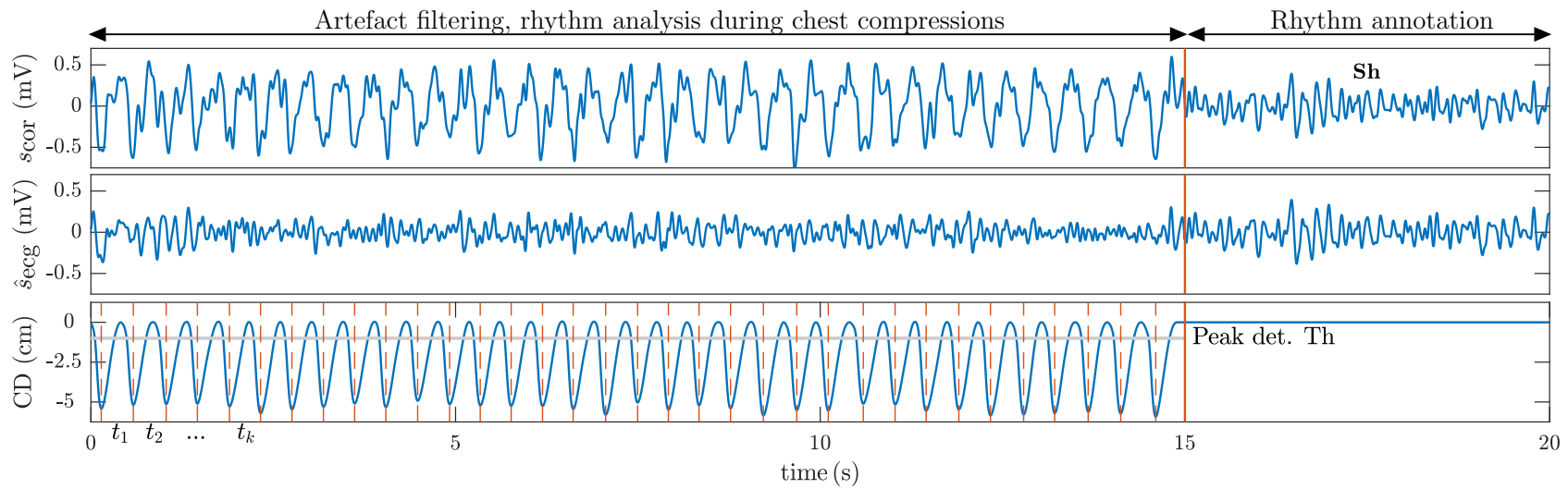

Figure 1. Example of a $20 \mathrm{~s}$ episode of the database. The top panel shows the ECG of a patient with a shockable rhythm (Sh): the first $15 \mathrm{~s}$ are corrupted by the CC artifact and the last $5 \mathrm{~s}$ are free of artifact showing the patient's underlying rhythm. The second pannel shows the filtered ECG and the bottom panel the CD signal with the CC instants $\left(t_{k}\right)$. 
For each filtered signal 244 features were computed [79] based on the multi-resolution analysis. The features were ranked by importance in each random forest (RF) classifier using the out-of-bag error [10]. For each set the top ranked 25 features were selected for classification.

\subsection{Classification}

The last step in the proposed SAA is classification. An ensemble of two RF classifiers were combined to reach a shock/no-shock decision, as can be shown in the last block of figure 2. The first classifier was trained using the selected 25 features from $\hat{s}_{\operatorname{ecg} \lambda_{1}}$ whereas the second one was trained using the selected 25 features from $\hat{s}_{\operatorname{ecg} \lambda_{2}}$. The final shock/no-shock decision was made based on the average value of the class posterior probabilities of two RF classifiers. The class with the higher average value of class posterior probabilities was chosen for shock/no-shock decision.

Both RF classifiers had 300 decision trees. Each tree was trained using bootstrapped replicas of the training data and the prior probabilities of each class (shock/no-shock) were balanced for each tree by using resampling. The cost function was defined to penalize the wrong diagnosis of nonshockable rhythms by a factor of $95 / 90$ based on the AHA recommendation.

\subsection{Model assessment}

A 10-fold cross-validation (CV) scheme was used to train and test the SAA. Folds were partitioned patient-wise ensuring that the rhythm prevalences matched to at least $85 \%$ the prevalences for shockable and nonshockable rhythms of the whole dataset (quasi-stratified).

Test segments were diagnosed as shock/no-shock based on the average value of class posterior probabilities (see section 2.4). These diagnoses were compared with the rhythm annotations to obtain the following performance metrics: Se, Sp and Balanced Accuracy (BAC), that is, the mean value of Se and Sp. In order to obtain the statistical distributions of these metrics the process was repeated 100 times. The results were compared to those obtained using the classical approach, filtering followed by a SAA designed for artifact-free ECG [11], in a single stage and multistage configurations.

\section{Results}

The mean (95\% confidence interval) $\mathrm{Se}, \mathrm{Sp}$ and BAC of the proposed double-stage SAA were $93.5 \%$ (92.9-94.0), 96.5\% (96.2-96.6) and 95.0\% (94.7-95.3), respectively. The classical approach in an optimal multistage configuration, as described in [5], yielded a Se, Sp and BAC of: $91.7 \%, 93.7 \%$ and $92.7 \%$, far below the obtained results using our proposed double-stage SAA.

A classical single stage solution produced an $\mathrm{Se}, \mathrm{Sp}$ and BAC of $96.3 \%, 81.3 \%$ and $88.8 \%$, respectively. The results for the best single RF-classifier $\left(\lambda_{2}\right)$ were $92.8 \%$ (92.3-93.5), 96.5\% (96.2-96.7) and 94.7\% (94.4-95.0), respectively. These results meet the minimum $90 \%$ Se and 95\% Sp performance goals recommended by the American Heart Association (AHA).

Table 1 shows the selected features for $\hat{s}_{\operatorname{ecg} \lambda_{1}}$ and for $\hat{s}_{\operatorname{ecg} \lambda_{2}}$, with the following notation: feature name (signal/wavelet coefficient). The first nine features of both columns are described by Figuera et al [7]. Features from 10 to 15 in the left column and from 10 to 12 in the right column were introduced by Rad et al [8]. Fuzzy Entropy (FuzzEn), the Signal Integral parameter (SignInt), the Peak Power Frequency (PPF), the Smoothed Nonlinear Energy Operator (SNEO) and the Hjorth Mobility parameter are described in [9, 12], [13], [14], [15] and [16], respectively. The remaining features were designed for this work: the number of QRS-like peaks (Npeak) and the Euclidean distance between the Hjorth Mobility and the Hjorth Mobility of the second degree (Mx2).

\section{Discussion}

This work introduces a double-stage SAA for a reliable rhythm analysis during CPR inspired by two solutions proposed in the literature to increase the Sp for rhythm analysis during CCs $[5,6]$. Our proposed SAA algorithm consists of a double-stage RLS filtering, multiresolution analysis for feature extraction, and two RF classifiers.

A single filtering stage followed by a commercial SAA yielded a Se and a Sp of $96.3 \%$ and $81.3 \%$ respectively. Using an ad-hoc SAA designed to diagnose filtered ECGs

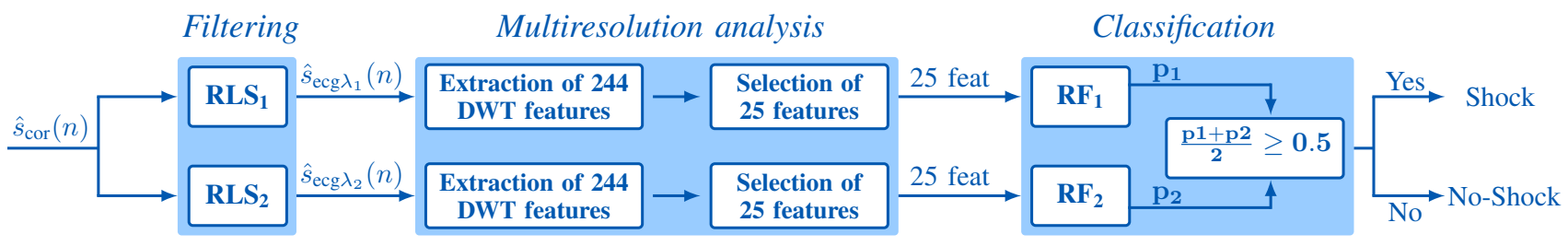

Figure 2. Arquitecture of the proposed double-stage SAA. 


\begin{tabular}{|c|c|c|c|}
\hline Feature & $\hat{s}_{\operatorname{ecg} \lambda_{1}}$ & Feature & $\hat{s}_{\operatorname{ecg} \lambda_{2}}$ \\
\hline 1 & $\mathrm{x} 1(s(n))$ & 1 & $\mathrm{x} 1(s(n))$ \\
\hline 2 & $\mathrm{x} 4(s(n))$ & 2 & $\mathrm{x} 4(s(n))$ \\
\hline 3 & $\operatorname{SamEn}\left(d_{3}\right)$ & 3 & $\operatorname{SamEn}\left(d_{3}\right)$ \\
\hline 4 & $\operatorname{SamEn}\left(s_{3}(n)\right)$ & 4 & $\operatorname{SamEn}\left(s_{3}(n)\right)$ \\
\hline 5 & $\operatorname{SamEn}\left(s_{4}(n)\right)$ & 5 & $\operatorname{SamEn}\left(s_{4}(n)\right)$ \\
\hline 6 & $\operatorname{vfleak}(s(n))$ & 6 & $\operatorname{vfleak}(s(n))$ \\
\hline 7 & count $2(s(n))$ & 7 & count $2(s(n))$ \\
\hline 8 & count $3(s(n))$ & 8 & $\mathrm{x} 3(s(n))$ \\
\hline 9 & $\mathrm{bCP}(s(n))$ & 9 & $\mathrm{bCP}(s(n))$ \\
\hline 10 & $\operatorname{IQR}(\dot{d}(5))$ & 10 & First Quartile $\left(d_{5}\right)$ \\
\hline 11 & $\operatorname{IQR}\left(d_{7}\right)$ & 11 & Positive Area $(s(n))$ \\
\hline 12 & $\operatorname{IQR}\left(\dot{d}_{7}\right)$ & 12 & Negative $\operatorname{Area}(s(n)$ \\
\hline 13 & $\operatorname{IQR}\left(\ddot{d}_{5}\right)$ & 13 & $\operatorname{Mean}\left(\dot{d}_{4}\right)$ \\
\hline 14 & $\operatorname{Var}\left(d_{5}\right)$ & 14 & $\operatorname{Mx}\left(d_{6}\right)$ \\
\hline 15 & $\mu_{2}\left(d_{7}\right)$ & 15 & $\operatorname{PPF}(s(n))$ \\
\hline 16 & $\operatorname{FuzzEn}(s(n))$ & 16 & $\operatorname{FuzzEn}(s(n))$ \\
\hline 17 & $\operatorname{FuzzEn}\left(s_{3}(n)\right)$ & 17 & $\operatorname{FuzzEn}\left(s_{3}(n)\right)$ \\
\hline 18 & $\operatorname{Mx} 2\left(\hat{s}_{\operatorname{ecg} \lambda_{1}}\right)$ & 18 & $\operatorname{FuzzEn}\left(s_{4}(n)\right)$ \\
\hline 19 & $\operatorname{SNEO}(s(n))$ & 19 & $\operatorname{SNEO}(s(n))$ \\
\hline 20 & $\operatorname{SignInt}\left(d_{7}\right)$ & 20 & $\operatorname{SignInt}\left(d_{7}\right)$ \\
\hline 21 & $\operatorname{SignInt}\left(d_{5}\right)$ & 21 & $\operatorname{Mean}(s(n))$ \\
\hline 22 & $\operatorname{Std}\left(\dot{d}_{3}\right)$ & 22 & $\operatorname{Std}\left(\dot{d}_{3}\right)$ \\
\hline 23 & $\operatorname{Mean}\left(d_{3}\right)$ & 23 & $\operatorname{Mean}\left(d_{3}\right)$ \\
\hline 24 & $\operatorname{Mean}\left(\dot{d}_{3}\right)$ & 24 & $\operatorname{Mean}\left(\dot{d}_{3}\right)$ \\
\hline 25 & Npeak $(s(n))$ & 25 & $\operatorname{Npeak}(s(n))$ \\
\hline
\end{tabular}

Table 1 . The 25 features selected by the two RF classifiers.

the Sp was increased in 15.2 points although the Se was reduced in 3.5 points. This significant increase in Sp allowed the AHA requirements to be met with an overall BAC of $94.7 \%$. The results were further increased with the addition of the double stage filtering, obtaining a BAC of $95.0 \%$.

In conclusion, this study confirms that ad-hoc decision algorithms for the filtered ECGs provide a reliable rhythm analysis during CPR and that the results would be further improved if the SAA combined the information derived from differently filtered ECG signals.

\section{Acknowledgements}

This work received financial support from the Spanish Ministerio de Economía y Competitividad, project TEC2015-64678-R jointly with the Fondo Europeo de Desarrollo Regional (FEDER); from UPV/EHU via GIU17/031 and from the Basque Government through grant PRE-2017-2-0137.

\section{References}

[1] Perkins GD, et al. European resuscitation council guidelines for resuscitation 2015: Section 2. adult basic life support and automated external defibrillation. Resuscitation 2015;95:81-99.

[2] Ayala U, Irusta U, Ruiz J, de Gauna SR, González-Otero D, Alonso E, Kramer-Johansen J, Naas H, Eftestøl T. Fully automatic rhythm analysis during chest compression pauses. Resuscitation 2015;89:25-30.
[3] Ruiz J, Ayala U, de Gauna SR, Irusta U, González-Otero D, Alonso E, Kramer-Johansen J, Eftestøl T. Feasibility of automated rhythm assessment in chest compression pauses during cardiopulmonary resuscitation. Resuscitation 2013; 84(9):1223-1228.

[4] Ruiz de Gauna S, et al. Rhythm analysis during cardiopulmonary resuscitation: past, present, and future. BioMed Research International 2014;2014.

[5] Isasi I, et al. A multistage algorithm for ECG rhythm analysis during piston driven mechanical chest compressions. IEEE Transactions on Biomedical Engineering 2018;

[6] Ayala U, et al. A reliable method for rhythm analysis during cardiopulmonary resuscitation. BioMed Research International 2014;2014.

[7] Figuera C, et al. Machine learning techniques for the detection of shockable rhythms in automated external defibrillators. PloS One 2016;11(7):e0159654.

[8] $\mathrm{Rad} \mathrm{AB}$, et al. ECG-based classification of resuscitation cardiac rhythms for retrospective data analysis. IEEE Transactions on Biomedical Engineering 2017; 64(10):2411-2418.

[9] Chicote B, et al. Application of entropy-based features to predict defibrillation outcome in cardiac arrest. Entropy 2016;18(9):313.

[10] Zabihi M, Rad AB, Katsaggelos AK, Kiranyaz S, Narkilahti $S$, Gabbouj M. Detection of atrial fibrillation in ecg hand-held devices using a random forest classifier. Computing 2017;44:1.

[11] Irusta U, Ruiz J, Aramendi E, de Gauna SR, Ayala U, Alonso E. A high-temporal resolution algorithm to discriminate shockable from nonshockable rhythms in adults and children. Resuscitation 2012;83(9):1090-1097.

[12] Chicote B, Irusta U, Aramendi E, Alcaraz R, Rieta J, Isasi I, Alonso D, Baqueriza M, Ibarguren K. Fuzzy and sample entropies as predictors of patient survival using short ventricular fibrillation recordings during out of hospital cardiac arrest. Entropy 2018;20:591.

[13] Wu X, et al. Signal integral for optimizing the timing of defibrillation. Resuscitation 2013;84(12):1704-1707.

[14] Eftestøl T, et al. Predicting outcome of defibrillation by spectral characterization and nonparametric classification of ventricular fibrillation in patients with out-of-hospital cardiac arrest. Circulation 2000;102(13):1523-1529.

[15] Chicote B, et al. Nonlinear energy operators for defibrillation shock outcome prediction. In Computing in Cardiology Conference (CinC), 2016. IEEE, 2016; 61-64.

[16] Gonzalez L, et al. Monitoring a skipped heartbeat: a real-time premature ventricular contraction (pvc) monitor. In Applications of Commercial Sensors (VCACS), 2016 IEEE Virtual Conference on. IEEE, 2016; 1-7.

Address for correspondence:

Name: Iraia Isasi Liñero

Full postal address: Ingeniero Torres Quevedo Plaza, 1, 48013

Bilbo, Bizkaia, Spain

E-mail address: iraia.isasi@ehu.eus 